I am pleased to have a copy, but the inclusion of many 'comparability' papers detracts. Their inclusion perhaps shows we have a long way to go in exploring - let alone usefully exploiting - the full potential of $\mathrm{X}$-ray/neutron complementarity. Tighter selection would have resulted in a slimmer volume, but one which would have been focused more on the central issues of complementarity. Space would then have been available for a useful editorial review to summarize the current state and encourage possible fruitful ways forward.

It is the reviewer's privilege to make a couple of minor gripes and I am no exception in asserting this right. Firstly, it would have helped finding my way through the book if each page had carried the running title of the appropriate chapter rather than that of the book. Secondly, I was just a little surprised to find some of my own unpublished results in print without my being asked. Perhaps if the results had been ready for publication, I wouldn't have minded so much ....

\section{Rutherford Appleton Laboratory}

J. L. FINNEY

Didcot

Chilton

Oxon $O X 110 Q X$

England

Acta Cryst. (1992). A48, 407

$X$-ray structure determination, a practical guide. By George H. Stout and Lyle H. Jensen. Pp. xv+ 453. John Wiley and Sons, New York, 1989. Price US \$44.00. ISBN 0-4716-0711-8.

The first edition of this book, published in 1968, was intended to enable the average chemist to perform crystal structure analyses for himself. Automatic data collection and structure-determination packages have made the experimental and computational aspects a great deal quicker and more routine than they were in 1968 , and it is tempting for the chemist/crystallographer to use them without much understanding of the processes or critical judgement. The authors would deplore such an approach and have provided a new edition of their book as a countermeasure. It covers a large amount of appropriate fundamental material, $\mathrm{X}$-rays, diffraction, unit cells and space groups and their determination, intensity measurements, structure factors and Fourier series, followed by structure solution and refinement. Every stage is carefully and thoroughly explained, with illustrative examples and many diagrams as well as a lot of practical detail and advice on strategy. For the chemist who is prepared to read the book purposefully or for anyone else doing single-crystal structure determination, there is enough information to carry out and understand every stage of a structure determination. Sometimes the detail is a little excessive: for example, a cell constant is derived from an oscillation film using an angle in radians and a second time using the angle in degrees; in the instructions for loading an oscillation camera it is suggested that, for practice, a first trial should use a dummy piece of film! In contrast, instructions for radiation safety, though some are given, are not adequate, at least by modern European standards. The presentation is one which will appeal to many chemists; equations are accompanied by an extensive explanation in words, rather than the more formal, elegant approach that is characteristic of a physicist's treatment.

The structure of the book is very similar to the earlier edition. The most notable exception is in the section on methods of solution of the phase problem; direct methods have, very properly, been given the first and most important position and their treatment expanded a little. A great deal of the earlier edition remains. In part this is a reminder that the fundamentals were all familiar 20 years ago - much of what has changed is in the implementation and the automation of the computations. In most chapters where it is relevant, new material has been added, rather concisely presented, together with useful references. The examples are mostly those of the earlier edition; they often provide good illustrations, but are probably not ones that would now be chosen on writing a new text book.

There are other more serious criticisms. It is not clear what kind of structures the book is meant to be most relevant to: the treatment is very good for small and medium-sized organic molecules; it is also appropriate for organometallics, but could be more helpful on problems associated with the presence of a few very heavy atoms (refinement strategies, residual peaks due to absorption errors etc). It discusses multiple isomorphous replacement, least-squares refinement with energy minimization, low-resolution refinement, all of which will normally only be relevant in protein studies, but it is not remotely adequate as a practical guide for protein crystallography. The treatment of anomalous dispersion is essentially unchanged since 1968 ; it is more relevant to proteins than to other types of stucture (since it assumes that structure amplitudes will normally be available for crystals with and without the anomalous scatterer present), but it is not presented in the form commonly used in protein crystallography. (Also there are discrepancies between terms used in the text, $F_{H}, \Delta F^{\prime}$, and in the figures, $f_{H}, \Delta f^{\prime}$, on, for example p. 302.) Oscillation, precession and Weissenberg photographs are described in detail, including both their fundamental geometry and practical use; diffractometers get much briefer treatment and some of the description is in terms of Weissenberg geometry, not the most helpful approach for today's chemists who may well meet a diffractometer before a Weissenberg camera. Also, it would be much more helpful if, on pp. 101-102, in relation to the orientation of crystals, all the diagrams had the goniometer head and spindle in the same orientation. In other parts too, the balance of material reflects the 1960 s rather than the 1990 s; for example, considerable space is devoted to refinement by differential Fourier series, which, to my knowledge, is not in current use. Misprints are few, but the quality of paper and print is poorer than in 1968 .

Despite these criticisms, the book does contain an immense amount of information, practical guidance and wisdom and is extremely readable. A copy of it should be available wherever a diffractometer system is generating structural data for chemists and it should help this process to be done with more understanding and judgement.
Department of Chemistry
University of Liverpool
Liverpool L69 3BX
England

MARJORIE M. HARDING 